\title{
DETERMINANTS OF POOR ANTHROPOMETRIC STATUS AMONG MOTHERS WITH CHILDREN 0-5 YEARS OLD
} IN THE PHILIPPINES

Eva Abille Goyena, Rovea Ernazelle G Austria, Midlred Ocampo Guirindola, Lynell Valdeabella Maniego, Cecilia Cristina Santos Acuin. DOST, FNRI, Taguig City, Philippines

\subsection{6/bmjopen-2015-forum2015abstracts. 87}

Background Poor maternal nutrition as indicated by chronic energy deficiency (CED) has been linked to a higher risk of dying due to pregnancy-related complications and is associated with negative birth outcome including intrauterine growth retardation that could lead to the intergenerational cycle of malnutrition.

Objectives Objective. This study aimed to identify the determinants of maternal under nutrition using data of the "2011 Updating Survey" conducted by FNRI-DOST.

Methods The 11,531 mothers with children 0-5 years old were included as participants and were classified into pregnant mothers, lactating mothers and non-pregnant/non-lactating mothers (NP/NL). The anthropometric measurements were assessed using the appropriate anthropometric indices for each physiologic group. Bivariate analysis using chi-square and binary logistic regression were applied to identify the potential determinants of maternal undernutrition.

Result The likelihood of undernutrition as indicated by CED among NPNL mothers increased with lower socioeconomic status (OR: 1.47; CI: 1.18-1.83), urban place of residence (OR: 1.25; CI: 1.04-1.49), younger age at first pregnancy (OR: 2.23; CI: 1.76-2.82), late timing of first prenatal visit (OR: 1.26; CI: 1.061.50), non-participation in prenatal services (OR: 1.31; CI: 1.09$1.56)$ and poor kitchen cleanliness (OR: 2.16; CI: 1.44-2.35). On the other hand, undernutrition among pregnant mothers is directly associated with 2 factors, the wealth status (OR: 2.19; CI: $1.27-3.79$ ) and consumption of variety of fruits (OR: 0.26 ; CI: $0.08-0.82$ ) while age was borderline odds. (OR: 0.45; CI: 0.18$1.07, p=0.07$ ). Among lactating mothers, the likelihood of undernutrition increased with longer duration of breastfeeding (OR: 1.86; CI: 1.40-2.47) while wealth status (OR: 1.28; CI: 0.99 1.67, $\mathrm{p}=0.06$ ), non- participation in postnatal services (OR: 1.27; CI: $0.98-1.66, p=0.07)$, non-consumption of calcium-rich foods (OR: 0.70 ; CI: $0.48-1.03, p=0.07$ ) and non-usage of food cover (OR: 1.94; CI: 0.99-3.81, $\mathrm{p}=0.05$ ) showed borderline odds.

Conclusion Maternal undernutrition is determined by socioeconomic, demographic, reproductive, health and dietary practices. Strategies aimed at improving maternal nutrition should prioritize (1) female adolescents, (2) schools and out-of-school youth programs, (3) gatekeepers in nutrition and health as part of support group team to ensure access to prenatal delivery and postnatal services. 